\title{
BREVES OBSERVAÇÕES SOBRE A TEORIA DAS REPRESENTAÇÕES SOCIAIS DE SERGE MOSCOVICI E A INTERDISCIPLINARIDADE
}

Cristiana Barcelos da Silva

Universidade Estadual Norte Fluminense

Gerson Tavares do Carmo

Universidade Estadual Norte Fluminense

\author{
Alessandra Maria Custódio da Silva
}

Universidade Estadual Norte Fluminense

\begin{abstract}
Resumo
O presente trabalho teve como objetivo analisar a Teoria das Representações Sociais (TRS) e a questão da Interdisciplinaridade enquanto categorias analíticas e alternativas metodológicas de produção científica. A partir de leituras inicias de autores basilares como, Moscovici (2003), Jodelet (2011, 1998), Sá (1998), Japiassu (1976), Fazenda $(1995,1979)$ e Frigotto (2008), dentre outros, verificamos algumas questões que faziam referência a respeito da possibilidade de interseção entre a TRS e a Interdisciplinaridade. Desse modo, este estudo buscou, com base em uma análise bibliográfica de cunho qualitativo, comprovar ou refutar a hipótese em torno da relação entre a teoria moscoviciana e a questão da Interdisciplinaridade. Importante destacar que, para a análise, levamos em conta a abordagem, os objetivos, os efeitos, bem como o contexto em que ambos se constituíram. Concluímos o estudo, afirmando a existência de conexão entre as duas temáticas, apontamos para quatro elementos característicos comuns: análise multifacetada de explicação da realidade, a dialogicidade, o foco no todo e a proposição em superar a fragilidade da fragmentação da ciência.
\end{abstract}

Palavras-chave: psicologia social; processos sociais; conhecimento.

\section{BRIEF COMMENTS ABOUT THEORY OF SOCIAL REPRESENTATIONS OF SERGE MOSCOVICI AND INTERDISCIPLINARITY}

\begin{abstract}
This study aimed to analyze the Social Representation Theory (SRT) and the issue of Interdisciplinarity as analytical categories and methodological alternatives of scientific production. From initial readings of authors such as basic, Moscovici (2003), Jodelet (2011, 1998), Sa (1998), Japiassu (1976), Finance (1995,1979) and Frigotto (2008), among others, we find some issues that made reference about the possibility of intersection between the SRT and the Interdisciplinary. Thus, this study, based on a literature review of qualitative nature, prove or disprove the hypothesis on the relationship between theory and moscovician the issue of interdisciplinarity. Importantly, for the analysis, we consider the approach, the objectives, the effects and the context in which they are constituted. Concluded the study by stating that there is connection between the two issues, we pointed to four common characteristic: multifaceted analysis of explanation of reality, dialogicity, focus on the whole and the proposition to overcome the weakness of fragmentation of science.
\end{abstract}

Keywords: social psychology; social processes; knowledge. 


\title{
BREVES COMENTARIOS SOBRE LA TEORÍA DE LAS REPRESENTACIONES SOCIALES DE SERGE MOSCOVICI Y LA INTERDISCIPLINARIEDAD
}

\begin{abstract}
Resumen
Este estudio tuvo como objetivo analizar la Teoría de las Representaciones Sociales (TRS) y la cuestión de la Interdisciplinariedad como categorías de análisis y alternativas metodológicas de la producción científica. A partir de las lecturas iniciales de autores como básico, Moscovici (2003), Jodelet (2011, 1998), Sa (1998), Japiassu (1976), Finanzas $(1995,1979)$ y Frigotto $(2008)$, entre otros, nos encontramos con algunos problemas que hace referencia a la posibilidad de intersección entre los TRS y la Interdisciplinario. Por lo tanto, este estudio, basado en una revisión de la literatura de naturaleza cualitativa, probar o refutar la hipótesis sobre la relación entre la teoría y moscoviciana el tema de la interdisciplinariedad. Es importante destacar que, para el análisis, consideramos el enfoque, los objetivos, los efectos y el contexto en que se constituyen. Concluido el estudio afirmando que no hay conexión entre los dos asuntos, señalamos a cuatro característica común: el análisis multifacético de la explicación de la realidad, dialogicidad, se centran en el todo y la proposición para superar la debilidad de la fragmentación de la ciencia.
\end{abstract}

Palabras clave: psicología social; los procesos sociales; conocimiento.

\section{INTRODUÇÃO}

A humanidade ao longo da história sempre procurou analisar e explicar os fenômenos naturais e sociais. Entretanto, na medida em que o conhecimento foi se tornando cada vez mais complexo, houve uma veemente necessidade de exigir uma análise integrada e multifacetada que explicasse a realidade. Fazendo referência à busca humana por saberes e sua relação com a vida social, o docente Gaudêncio Frigotto (2008) argumentou o seguinte:

O homem, na busca incessante de satisfazer suas múltiplas e sempre históricas necessidades de natureza biológica, intelectual, cultural, afetiva e estética, estabelecem as mais diversas relações sociais. A produção do conhecimento e sua socialização ou negação para determinados grupos ou classes não é alheia ao conjunto de práticas e relações que produzem os homens num determinado tempo e espaço (Frigotto, 2008, p. 43).

Nesse contexto, Hobsbawn (1962), desejando compreender como e o porquê do mundo vira ser o que é bem como para onde se dirige, explicou em seu livro "A Era das revoluções" que o século XX foi marcado por inúmeras transformações, sobretudo do ponto de vista da ciência, da filosofia, da religião e da arte e, por conseguinte, dos impactos produzidos por essas mudanças.

O fato de pensar na história da humanidade e no que tratou o pesquisador foi que nos estimulou a refletir nesse trabalho, sobre a existência ou não de uma relação entre a questão da Interdisciplinaridade e a Teoria das Representações Sociais (TRS) mostrando suas potencialidades metodológicas no campo da produção científica interdisciplinar. 


\section{MÉTODO A: REVISÃO DE LITERATURA}

\section{O CONCEITO DE INTERDISCIPLINARIDADE}

Ao elaborar "O Dicionário latino-português", Saraiva (2006, p. 45) faz referência ao vocábulo "inter" e explicou que o termo esteve relacionado à preposição "entre (indicando separação reciprocidade), entre o número de, no meio de".

Silva Thiesen (2008) revelou que, em termos gerais, a discussão sobre a temática da Interdisciplinaridade tem sido tratada por duas diferentes abordagens, uma de natureza epistemológica e outra de natureza pedagógica. A primeira, tomando como categoria analítica, o estudo do conhecimento e seus aspectos de socialização, produção e reconstrução do conhecimento; a ciências e seus modelos de investigação; e o método compreendido como mediador que intermedia sujeito e realidade. O outro seria o enfoque pedagógico tratando de questões de caráter curricular e, por conseguinte do processo de ensinoaprendizagem.

Ainda segundo Silva Thiesen (2008), independente da categoria de análise, a literatura tem demonstrado que há uma característica comum e consensual quanto à finalidade da interdisciplinaridade: se apresentou como alternativa que procurou responder as necessidades de superação do olhar fragmentado presente nos processos de produção e socialização do conhecimento. Versou, portanto, de um movimento, sobretudo nas Ciências Humanas e Sociais que caminhou para novas formas de organização do conhecimento ou para um novo sistema que propôs novas posturas frente a sua produção, difusão e transparência. Como exemplos desse movimento podemos citar as obras de Snow (1959), Kapp (1961), Gustodorf (1967), Piaget (1973), Vygotski (1986) e Durand (1991).

Elaborando um recorte histórico, um estudo de Alvarenga, Phillip, Sommerman, e Ams (2010) afirmou que a Interdisciplinaridade, teve suas raízes na ciência moderna, sobretudo aquela produzida a partir do século XX. Contudo, para compreendê-la, pareceu necessário apontar para o início dos estudos científicos que dataram do século $\mathrm{XV}$, quando a sociedade passou por uma mudança estrutural e que resultou numa explosão de novos conhecimentos, novas práticas e novas técnicas de pesquisa. Surgiu, definitivamente, a ciência e a pesquisa científica com a missão de apresentar a razão em oposição à fé e a pesquisa em oposição ao discurso e a retórica.

Na visão de Fazenda (1995), referindo-se a um movimento, a um conceito e a uma prática em processo de construção e desenvolvimento dentro das ciências, a Interdisciplinaridade buscou definir um objeto também em construção: a sociedade. Não qualquer sociedade, mas aquela que se autoexplicava de forma compartimentada, a qual a disciplinarização, entendida como 
"fatias" dos estudos científicos e das disciplinas escolares como matemática, biologia, ciências naturais, história, e outras, tentavam dar contas dos fenômenos naturais e sociais. Desse modo, foi de um esforço em sobrepujar o conceito de disciplina e suas limitações que a Interdisciplinaridade nasceu.

Por essa perspectiva, Alvarenga et al. (2010) explicaram que, em termos de prática de pesquisa, a aplicação da Interdisciplinaridade na ciência impulsionou, a partir do século $X X$, o desenvolvimento de novas práticas de pesquisa, com o propósito de unir as disciplinas, para que juntas tentassem produzir respostas aos problemas.

A Interdisciplinaridade, como um enfoque teórico-metodológico ou gnosiológico, denominada por Gadotti (1999), insurgiu na segunda metade do século XX em resposta a uma necessidade verificada principalmente nos campos das Ciências Humanas, buscando superar a fragmentação e o caráter de especialização do conhecimento propostos por uma epistemologia de tendência positivista cujas raízes eram representadas pelo empirismo, naturalismo e o cientificismo do início da modernidade. Afirmou o autor:

A interdisciplinaridade, como questão gnosiológica, surgiu no final do século passado, pela necessidade de dar uma resposta à fragmentação causada por uma epistemologia de cunho positivista. As ciências haviam-se dividido em muitos ramos e a interdisciplinaridade restabelecia, pelo menos, um diálogo entre elas, embora não resgatasse ainda a unidade e a totalidade (Gadotti, 1999, p.1).

Segundo Silva Thiesen (2008), grandes nomes da ciência moderna como Descartes, Bacon, Galileu, Nilton e Darwin influenciaram de certa maneira a divisão da ciência em áreas de conhecimento, estimulando sua especialização cada vez mais intensa e focada. As chamadas correntes naturalistas e mecanicistas procuravam a partir do período da Renascença, estabelecer uma concepção de mundo mais científica. Nesse contexto, foi que a Interdisciplinaridade enquanto movimento no período contemporâneo nasce com vista a uma perspectiva de estímulo ao diálogo e interação do conhecimento, na tentativa " $(. .$.$) de romper como o caráter de hiperespecialização e com a$ fragmentação dos saberes (p. 546)".

O pesquisador Goldman (1979) ao discutir dialética e cultura, acrescentou que trabalhar de forma interdisciplinar, significava compreender melhor a relação entre o todo e as partes que a compõem. Reconheceu as contribuições das formulações do materialismo histórico para as discussões futuras do conceito de Interdisciplinaridade no âmbito científico.

Para Silva Thiesen (2008), apesar de parecer um conceito púbere e algumas pesquisas se apresentarem muito recentes no Brasil, o conceito de Interdisciplinaridade chegou por meio dos estudos de Georges Gusdorf (sobretudo de sua obra denominada La Parole publicada em 1963) e Piaget. No país, autores como Ivani Fazenda e Hilton Japiassu se destacaram quanto à 
produção de trabalhos sobre o tema. Fazenda influenciado por Gusdorf e Japiassu por Piaget.

A pesquisadora Ivani Fazenda (1979) vinculou suas publicações à questão da educação, voltando seus estudos para a evolução histórico-crítica do conceito e para o retrato do seu nascimento e desenvolvimento.

Já o estudioso Hilton Japiassu (1976) vem discutindo o movimento contemporâneo presente nas dimensões da epistemologia e da pedagogia que marcaram o rompimento com a visão cartesiana e mecanicista de mundo, e assumiram uma concepção integradora, dialética e totalizadora na construção do conhecimento via Interdisciplinaridade. Para ele, uma das características da Interdisciplinaridade seria o fato de propor duas questões: as trocas entre as especialidades e um processo de integração real das áreas de conhecimentos em torno de um mesmo projeto.

Assim, em relação à definição de conceitos, metodologias e caminhos para a Interdisciplinaridade, tudo pareceu estar em processo de contínua construção. O que pareceu estar claro, porém, foi a ideia de que o conceito significara uma alternativa de superação da compartimentalização da ciência, como afirma Silva Thiesen (2008):

(...) Independente da definição que cada autor assuma, a interdisciplinaridade está sempre situada no campo onde se pensa a possibilidade de superar a fragmentação das ciências e dos conhecimentos produzidos por elas e onde simultaneamente se exprime a resistência sobre um saber parcelado (p. 547).

Alvarenga et al. (2010) alegaram que, após o século $X X$, as pesquisas interdisciplinares se justificaram por várias razões, dentre elas, por propor uma análise multifacetada dos fenômenos, assim como o diálogo entre as diversas áreas do conhecimento, por estimular o foco no todo e por ter a disciplinarização da ciência apresentado inúmeras limitações. Desse modo, a Interdisciplinaridade surgiu, portanto, como proposta de construção, de certo modo, mais holística de construção do conhecimento.

Como resultado desse movimento, a aplicação da Interdisciplinaridade na ciência recomendou também, o surgimento de novas disciplinas que unissem áreas específicas a fim de compreender fenômenos que seriam incompreensíveis com o conhecimento de apenas um campo de conhecimento. Como um dos exemplos dessas disciplinas, chamadas agregadoras (por já nascerem estruturalmente de forma híbrida, ou seja, da junção de mais de uma área de conhecimento), podemos citar a Psicologia Social e como uma das suas ramificações, a TRS enquanto subproduto e categoria epistemológica que em sua origem recebeu influência tanto da Psicologia quanto da Sociologia. 


\section{MÉTODO B: REVISÃO DE LITERATURA}

\section{A TEORIA DE REPRESENTAÇÕES SOCIAIS DE MOSCOVICI}

Se nos atentarmos para a origem etimológica da palavra "representação", veremos que veio do latim, inicialmente "representare", significando fazer presente, apresentar de novo. Em um trabalho chamado "História e representação", o pesquisador Falcon (2000) explicou que representar significava, portanto, fazer presente uma ideia, algo ou alguém.

Jovchelovitch (1998) em seus estudos apontou que a expressão representações sociais foi mencionada pela primeira vez em 1961 pelo romeno Serge Moscovici. Em seu estudo sobre a representação social da psicanálise que recebeu o título de "Psychanalyse: son image et son public", Moscovici apresentou um trabalho que tentava considerar as representações sociais não como um conceito, mas como um fenômeno. Ao definir o verbo representar, o teórico pioneiro na formulação da TRS escreveu o seguinte:

Representar significa a uma vez e ao mesmo tempo, trazer presente as coisas ausentes e apresentar coisas de tal modo que satisfaçam as condições de uma coerência argumentativa, de uma racionalidade e de uma integridade normativa do grupo. É, portanto, muito importante que isso se dê de forma comunicativa e difusiva, pois não há outros meio, com exceção do discurso e dos sentidos que eles contêm, pelos quais as pessoas e os grupos sejam capazes de se orientar e se adaptar as tais coisas (Moscovici, 2013, p. 216).

Em um de seus trabalhos, Alexandre (2004) esclareceu que o termo representações sociais, como ferramenta da Psicologia Social nasceu a partir dos estudos de Moscovici que negou a dicotomia existente entre o individual e o social, até então representadas respectivamente pela Psicologia que se encarregava de explicar o sujeito na sua individualidade e pela Sociologia com seus estudos sobre a sociedade, no seu coletivo.

Nas palavras de Moscovici (2013), as representações sociais teriam como característica peculiar o senso comum coletivo, uma vez que buscam analisar

(...) aqueles modos de pensamentos que a vida cotidiana sustenta e que são historicamente mantidos por mais ou menos longos períodos; modos de pensamentos aplicados a objetos diretamente socializados, mas que, de maneira cognitiva e discursiva, as coletividades são continuamente orientadas a reconstruir nas relações de sentido aplicado à realidade e a si mesmo (p. 208).

A TRS, desenvolvida e apresentada por Moscovic (2013), tratou das produções dos saberes sociais, centrando-se na análise da construção e transformação do conhecimento social e elucidação da forma como a ação do pensamento individual se interligava na dinâmica social. Imprescindível ressaltar que, nessa conjectura, os saberes alvo eram aqueles que se produziam no cotidiano e que pertenciam ao mundo vivido. 
Sob a ótica de Jodelet (2011), um dos motivos que mais levou Moscovici a desenvolver o estudo das representações sociais, dentro de uma metodologia científica, foi sua crítica aos pressupostos positivistas e funcionalistas das demais teorias que não explicavam a realidade sob diversas dimensões. A respeito da natureza diversa da teoria, a seguidora de Moscovici (2013), discorreu, numa palestra proferida no Brasil, o seguinte:

Entre as razões que explicam a força desse movimento, já mencionei o alcance da Teoria das Representações Sociais. A diversidade das dimensões psíquicas, intelectuais e cognitivas que ela abraça, a diversidade dos níveis sociais, individuais, interinindividuais, intergrupais e ideológicos que ela articula permitem dar conta da complexidade dos fenômenos que uma psicologia autenticamente social deve considerar (p. 21).

Para Duveen (2013), a complexidade dos indivíduos e dos fenômenos sociais, acabaram por motivar Moscovici e seus seguidores, a buscarem via TRS, construir uma ponte entre os conceitos sociológicos e os psicológicos. O pesquisador da Grã-Bretanha ao prefaciar o livro do prenunciador da TRS escreveu o seguinte: "(...) a Teoria das Representações Sociais de Moscovici procurou tanto reconhecer um fenômeno social específico, como fornecer os meios para torná-lo inteligível como um processo sociopsicológico" (p. 28).

A respeito da transparência e da natureza ampla da teoria, o próprio Moscovici (2013) esclareceu o seguinte: "Seja como for, a aspiração da teoria das representações sociais é clara, pelo fato de assumir como seu centro a comunicação e as representações. A teoria espera elucidar os elos que unem a psicologia humana com as questões culturais contemporâneas" (p. 206).

Reforçando a ideia do autor supra citado, Jodelet (2011), ao elucidar que no plano epistemológico a TRS se apresentava como resolução aos estudos humanos centrados em processos intraindividuais, explanou a seguinte ideia: "Ela restitui ao pensamento e aos processos psíquicos seu caráter dialógico. Considerando os contextos em que se inscrevem a prática e a ação, ela reintroduz, na análise dos fenômenos representativos, a ordem da cultura e a da história" (p. 21).

Quanto à dimensão funcional da teoria, enquanto produto social e aporte para compreender a realidade, o francês Jean-Claude Abric (1998) que estuda a representação no mundo do trabalho revelou o seguinte:

A representação funciona como um sistema de interação da realidade que rege as relações dos indivíduos com seu meio físico e social, ela vai determinar seus pensamentos e suas práticas. A representação é uma guia para a ação, ela orienta as ações e as relações sociais. Ela é um sistema de pré-decodificação da realidade por que ela determina um conjunto de antecipações e expectativas (p. 28).

A análise de Sá (1998) revelou, que a busca por tratar o indivíduo e a tentativa de abarcar sua complexibilidade talvez retratasse algum dos pontos que expliquem a visão holística das representações e, por conseguinte, desse 
campo de pesquisa que abrangeu pesquisas de diversas amostragens temáticas. Ao analisar a noção de representação social com enfoque na educação, partindo de um questionamento esclareceu o seguinte:

Quais são os problemas ou fenômenos de representação social que têm sido preferencialmente explorados? A rigor, a diversidade de problemas pesquisados é tão grande que se corre o risco de sua apresentação parecer uma espécie de 'catálogo de supermercado'. Tentaremos atenuar essa impressão, agrupando os numerosos problemas em sete temas substantivos gerais, que parecem configurar áreas mais consistentes de interesse de pesquisadores: ciência, saúde, desenvolvimento, educação, trabalho, comunidade e exclusão social (p. 34).

A pesquisadora Jovchelovitch (1998), se referindo à grande teoria, como denominam os especialistas da área, entendeu-a como uma teoria sobre os saberes sociais produzidos na vida cotidiana e originados por ela, pela construção e transformação dos saberes sociais em relação a diferentes contextos. Garantiu que a relação entre o saber, a vida e o contexto indicou para a necessidade de atenção e compreensão das várias formas assumidas pelo conhecimento e as racionalidades que a mantêm, ou seja: a teoria se referiria tanto ao processo pelo qual são elaboradas as representações quanto como às estruturas do conhecimento que são estabelecidas. Nessa perspectiva, a TRS esteve, na visão da autora, "preocupada em compreender como pessoas comuns, comunidades e instituições produzem saberes sobre si mesmos, sobre os outros e sobre a multidão de objetos sociais que lhes são relevantes" (p. 87).

Na visão de Sá (1998), notório ressaltar a importância e veemência da TRS enquanto campo complexo e interdisciplinar de estudos, por conta de algumas de suas características, pois propôs uma análise complexa dos fenômenos humanos, bem como a dialogicidade entre os campos da Psicologia e da Sociologia, estimulando a pesquisa no todo do ser humano enquanto indivíduo que se produz no social, tentando assim, superar a dicotomia dos estudos entre o individual e o coletivo.

Tamanha sua potencialidade e sugestões metodológicas na construção da ciência, percebemos uma expansão nos países, a apropriação da TRS, uma vez que "o campo de estudo das representações sociais se encontra em franca expansão no Brasil, não apenas no âmbito da psicologia social, mas também nos de disciplinas aplicadas, como educação, enfermagem e serviço social [...]" (Sá, 1998 , p. 15).

\section{RESULTADOS: OBSERVAÇÕES RELACIONAIS ENTRE A TEORIA DAS REPRESENTAÇÕES SOCIAIS E A INTERDISCIPLINARIDADE}

Se atentarmos para o significado da palavra Interdisciplinaridade, perceberemos que o sentido denotativo apresentado na primeira parte desse trabalho, fez referência à ideia de ponte, de lugar intermediário entre uma 
"coisa" e outra. Por outro lado, se tratando da TRS, esta pareceu fazer parte, do ponto de vista estrutural, de um constructo dialógico a partir da intermediação entre a área da Psicologia e área da Sociologia. Moreira e Oliveira (1998), ao explicitarem a interface entre Interdisciplinaridade e representações sociais, partiram dessa noção de diálogo entre as disciplinas, explicando assim, sua particularidade de natureza interdisciplinar e o estabelecimento de comunicação em níveis de contribuição e entendimento entre as disciplinas, de forma a auxiliar a compreensão cada vez mais clara dos objetos de pesquisa (Moreira \& Oliveira, 1998).

Por essa perspectiva, Alves, Brasileiro e Brito, (2004), em um trabalho que abordou de forma crítica e histórica a Interdisciplinaridade enquanto conceito em construção explorou o potencial das representações sociais de explicar os fenômenos sociais em um enfoque dialético, compreendendo o homem com ser psicológico e social. Sobre o caráter interdisciplinar da teoria moscoviciana, nos escritos da estudiosa observou-se o seguinte:

Uma pista para o entendimento dessa teoria como interdisciplinar sinaliza para o campo epistemológico, mais especificamente a superação da dicotomia sujeito/objeto. Significa dizer que os múltiplos constructos e possibilidades metodológicas, permitem a construção dos objetos de pesquisa sobre a base da integração entre as pessoas e os processos sociais. Talvez seja este o caminho a ser percorrido na direção da explicitação do caráter interdisciplinar dessa teoria (p. 21).

Nas palavras de Alves et al. (2004), em termos práticos, Interdisciplinaridade e TRS, ambos partiram de um empenho na busca por superar a fragmentação do conhecimento, relacionando realidade e os problemas da vida moderna. No âmbito científico, os esforços em prol da elaboração e explicação de ambas as teorias, estariam atreladas ao empenho de perseguir respostas, impossíveis de serem alcançadas, com os conhecimentos fragmentados de uma única área especializada.

Pensando na TRS, Jodelet (1998), argumentou que ela, por natureza, transita em todas as Ciências Humanas. Possui, portanto, uma origem híbrida e uma essência interdisciplinar, enquanto conceito articulador das múltiplas perspectivas de diversos campos de pesquisa, permitindo, sobretudo uma interface entre noções psicológicas e sociológicas.

Ao se posicionar em relação a ambos os campos de conhecimento, Sá (1998) se reportou a importância de eventos vivenciados no Brasil como o Encontro Nacional sobre Representação Social e Interdisciplinaridade, ocorrido em julho de 1997 em João de Pessoa e o IX Encontro Nacional de Psicologia Social realizado em setembro do mesmo ano em Belo Horizonte. O autor pareceu tecer seu discurso, considerando o movimento no país de estímulo, dedicação e esforços de novas e consistentes pesquisas, que tomassem os dois campos enquanto suportes teórico-metodológicos e conceituais. 


\section{DISCUSSÃO}

O presente trabalho objetivou refletir sobre a TRS e a Interdisciplinaridade enquanto potenciais metodológicos na construção do conhecimento científico.

Constatamos que a Interdisciplinaridade emergiu enquanto termo científico com a pretensão de superar algumas fragilidades da fragmentação da ciência e como uma resposta à necessidade de reconciliação epistemológica e social na produção do conhecimento. Percebemos, ao longo das análises, o quanto a complexidade do conhecimento exigiu historicamente uma análise integrada, holística e multifacetada de explicação da realidade.

Percebemos que a TRS tratou das produções dos saberes sociais, centrando-se na análise da construção do conhecimento social, elucidando formas explicativas de compreender como a ação do pensamento individual se conectava a dinâmica das relações sociais.

Nessa perspectiva, tanto a Interdisciplinaridade, como a TRS, emergiram enquanto categorias de análises, com vista a inovadoras maneiras de fazer ciência, reconhecendo a complexidade do conhecimento, do ser humano e a necessidade de superação da fragmentação da ciência.

Se tratando dessas relações, impossível não compreender a TRS e a Interdisciplinaridade como categorias analíticas de natureza integral e multifacetada, na medida em que, demandam práticas de produção de conhecimento condizentes com sua natureza, no sentido de abarcar ao máximo a totalidade.

Sendo assim, este estudo buscou suscitar algumas discussões envolvendo a TRS e a Interdisciplinaridade, considerando que ambas marcaram um movimento de superação da dimensão unilateral e disciplinar do conhecimento, trazendo para a ciência, formas alternativas, complementares e inovadoras para o processo de conhecer.

\section{REFERÊNCIAS}

Abric, J. C. (1998). A abordagem estrutural das representações sociais. Estudos interdisciplinares de representação social, 2, 27-38.

Alexandre, M. (2004). Representação social: uma genealogia do conceito. Comum, 10(23), 122-38.

Alvarenga, A. D., Phillipi JR, A., Sommerman, A. A., \& Ams, F. (2011). Histórico, fundamentos filosóficos e teórico-metodológicos da interdisciplinaridade. In A. Philipi Jr \& A. J. S. Neto (Eds.), Interdisciplinaridade em ciência, tecnologia \& inovação (3-68). Barueri, SP: Manole.

Alves, R. F., Brasileiro, M. D. C. E., \& Brito, S. M. D. O. (2004). Interdisciplinaridade: um conceito em construção. Episteme, 19(02). 
Duveen, G. (2013). Prefácio. In: In S. Moscovici (Ed.), Representações sociais: Investigações em psicologia social. Petrópolis, RJ: Vozes.

Fazenda, I. C. A. (1979). Integração e interdisciplinaridade no ensino brasileiro (Vol. 4). São Paulo, SP: Edições Loyola.

Fazenda, I. C. A. (1995). Interdisciplinaridade: História, teoria e pesquisa. Campina, SP. Papirus Editora.

Frigotto, G. (2008). A interdisciplinaridade como necessidade e como problema nas ciências sociais. Ideação, 10(1), 41-62.

Gadotti, M. (1999). Interdisciplinaridade: atitude e método. São Paulo: Instituto Paulo Freire. Recuperado de http://ftp-acd.puccampinas.edu.br/pub/professores/cchsa/lucianeoliveira/Planejamento\%20d e\%20Ensino/Tema\%202\%20-\%20Interdisciplinaridade/Texto\%202\%20$\% 20$ Interdisciplinaridade.pdf

Hobsbawm, E. J. (1962). A era das revoluções (Vol. 4). São Paulo: Paz e terra. Japiassu, H. (1976). Interdisciplinaridade e patologia do saber. Rio de Janeiro: Imago.

Jodelet, D. (2011). Conferência de Denise Jodelet por ocasião do recebimento do título de doutor Honoris Causa da Universidade Federal do Rio de Janeiro. In A. J. Mazzotti (Ed.), Representações sociais: aspectos teóricos e aplicações à educação. Múltiplas Leituras, 1(1), 18-43.

Jodelet, D. (1998) Prefácio. In C. P. Sá (Ed.), A construção do objeto de pesquisa em representações sociais. Rio de Janeiro: EdUERJ.

Jovchelovitch, S. (1998). Representações Sociais: para uma fenomenologia dos saberes sociais. Psicologia e Sociedade, 10(1), 54-68.

Moreira, A.S.P. \& Oliveira, D.C. (1998). Apresentação. In A.S.P. Moreira \& D.C. Oliveira (Eds.), Estudos interdisciplinares de representações sociais. Goiânia: AB.

Moscovici, S. (2013). Representações sociais: Investigações em psicologia social. Petrópolis: Vozes.

Sá, C. P. (1998). A construção do objeto de pesquisa em representações sociais. Rio de Janeiro: EdUERJ.

Saraiva, F. D. S. (2006). Novíssimo dicionário latino-português. Etimológico, prosódico, histórico, geográfico, mitológico, biográfico, etc. Rio de Janeiro: Garnier.

Silva Thiesen, J. (2008). A interdisciplinaridade como um movimento articulador no processo ensino-aprendizagem. Revista Brasileira de Educação, 13(39), 545-554. 
Sobre os autores

Cristiana Barcelos da Silva é pedagoga, professora de Educação Básica e mestranda do Programa de Mestrado em Cognição e Linguagem.

cristianabarcelos@yahoo.com.br

Gerson Tavares do Carmo é doutor em Sociologia Política pela Universidade Estadual do Norte Fluminense, professor associado do Laboratório de Estudos de Educação e Linguagem (LEEL) do curso de Licenciatura em Pedagogia e dos Programas de Pós-graduação em Cognição e Linguagem e Políticas Sociais (UENF).gtavares33@yahoo.com.br

Alessandra Maria Custódio da Silva é professora de Educação Básica e mestranda do Programa de Mestrado em Cognição e Linguagem (UENF).

alessandrapsiu@yahoo.com.br

Recebido em: 18/12/2014

Revisado em: 17/06/2015

Aceito em: $23 / 10 / 2015$ 